\title{
Dust concentration and soil properties at the TMT candidate sites
}

S. G. Els, R. Riddle, M. Schöck, W. Skidmore, T. Travouillon

S. G. Els, R. Riddle, M. Schöck, W. Skidmore, T. Travouillon, "Dust concentration and soil properties at the TMT candidate sites," Proc. SPIE 8444, Ground-based and Airborne Telescopes IV, 844468 (17 September 2012); doi: 10.1117/12.926863

Event: SPIE Astronomical Telescopes + Instrumentation, 2012, Amsterdam, Netherlands 


\title{
Dust concentration and soil properties at the TMT candidate sites
}

\author{
S.G. Els ${ }^{a, b, c}$, R. Riddle ${ }^{c, d}$, M. Schöck ${ }^{c}$, W. Skidmore ${ }^{c}$, T. Travoullion ${ }^{c}$ \\ ${ }^{a}$ Gaia DPAC, c/o ESAC, PO Box 78, 28691 Villanueva de la Cañada, Spain \\ ${ }^{b}$ Cerro Tololo Inter-American Observatory, Casilla 603, La Serena, Chile \\ ${ }^{c}$ TMT Observatory Corporation, 2632 E. Washington Blvd., Pasadena, CA 91107, USA \\ ${ }^{d}$ Caltech Optical Observatories, Caltech, 1200 E. California Blvd, Pasadena, CA 91125, USA
}

\begin{abstract}
During the site testing campaign for the Thirty Meter Telescope (TMT) in addition to the optical conditions of the atmosphere, measurements of the soil surface properties were obtained also. The dust concentration in the air was measured by means of dust sensors which were mounted underneath the mount of the site monitoring telescopes. The ground heat fluxes and soil temperatures were measured several centimeters into the ground. On Cerro Armazones it was also possible to conduct an experiment to measure heat conduction of the soil. In this paper, all of these measurements are described, the results and their potential use is summarized.
\end{abstract}

Keywords: Site testing, atmospheric conditions, dust

\section{INTRODUCTION}

Ground based astronomical observatories are exposed to their terrestrial environment. While the atmospheric conditions are commonly monitored as prime indicators of the quality of any potential observatory site, the soil will also affect astronomical observations. Dust particles in the atmosphere increase the opacity, thus dimming star light, as well as producing scattering which will enhance potential light pollution and the sky background. Experiences with these effects reported in the literature showed that they depend on the specific dust and meteorological properties and therefore differ from site to site ${ }^{(1,2,3)}$.

Aside from such optical effects, the operation of astronomical telescopes during times with high dust content can cause dust accumulating on the optical surfaces, lowering the overall system throughput. Also sensitive measurement equipment can be affected by the accumulation of dust particles on sensitive areas. ${ }^{4}$ It is therefore recommendable that site testing projects also assess the dust concentration in the atmosphere either by remote sensing techniques, ${ }^{5}$ and/or by using in-situ measurements.

Here the aspects of dust and soil measurements during the site testing for the Thirty Meter Telescope (TMT) are presented. TMT investigated a set of five candidate sites by means of a thorough site testing campaign for their suitability to host an extremely large telescope (ELT) such as TMT. ${ }^{6}$ The site monitoring equipment also included dust, soil temperature and heat flux sensors at each of the candidate sites. The five mountains which were investigated by the TMT sites program were: Cerro Tolar $(2290 \mathrm{~m})$, Cerro Armazones (3064 m), Cerro Tolonchar $(4480 \mathrm{~m})$ which are located in Northern Chile, San Pedro Mártir $(2830 \mathrm{~m})$ in Baja California in Mexico, and 13N (4050 m) on Mauna Kea, Hawaii, USA.

This paper reports in Section 2 on the instrumentation and an initial assessment of the dust measurements as conducted by the TMT site testing project.

Also, during the final stages of the TMT site testing project, an experiment was setup to assess the energy properties of the soil at Cerro Armazones. Currently, the heat conductivity coefficient is the parameter which could be determined. Details of this experiment and first results are reported in Section 3.

Finally, a summary is given in Section 4.

Further author information: (Send correspondence to S.G.E.)

S.G.E.: E-mail: sels@sciops.esa.int

Ground-based and Airborne Telescopes IV, edited by Larry M. Stepp, Roberto Gilmozzi, Helen J. Hall,

Proc. of SPIE Vol. 8444, 844468 - (c) 2012 SPIE · CCC code: 0277-786/12/\$18 - doi: 10.1117/12.926863 


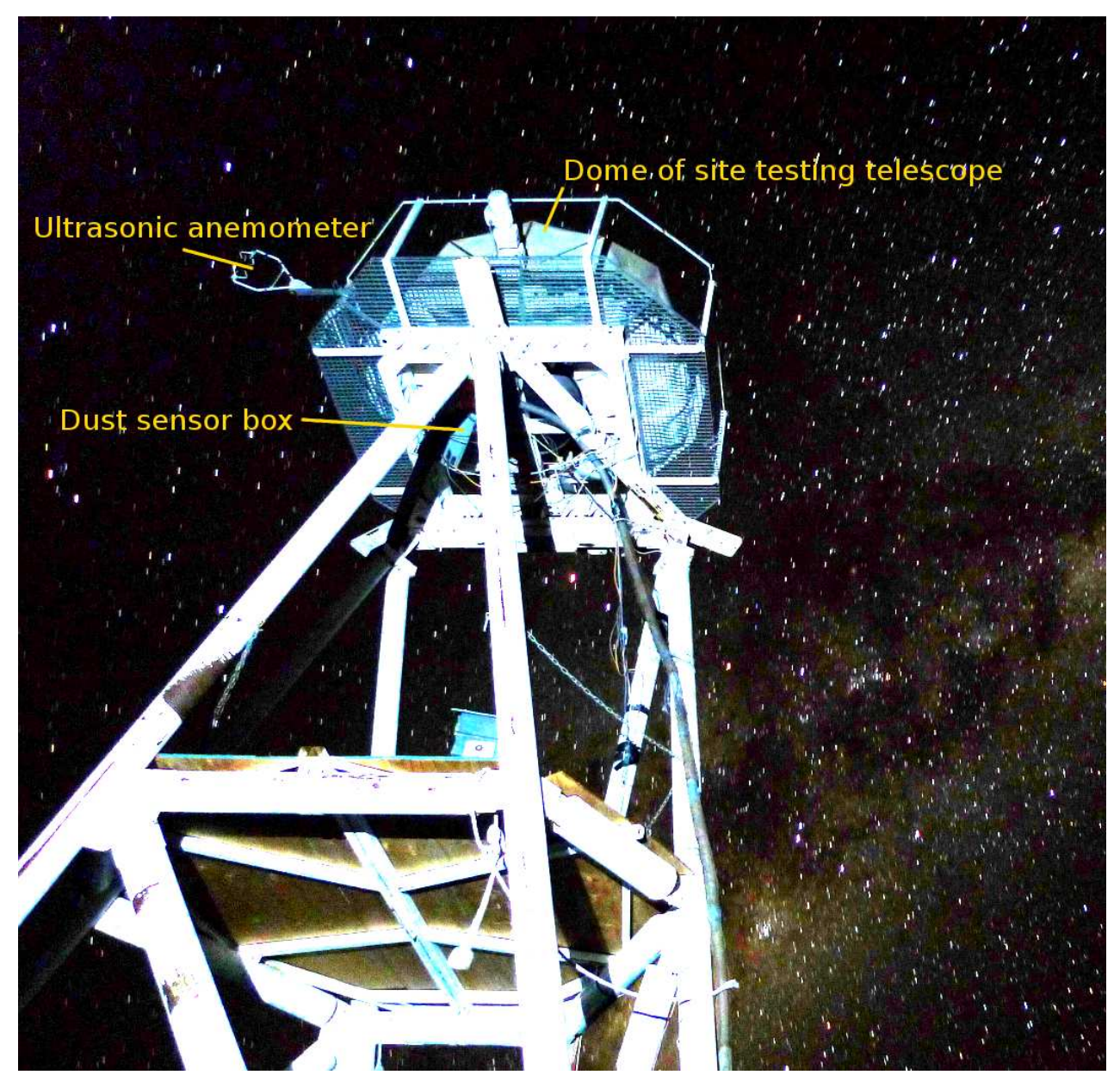

Figure 1. (color online) Night time image of a TMT site testing telescope on its tower. The location of the telescope dome and main instruments which were deployed on those towers are indicated.

\section{DUST CONCENTRATION IN THE ATMOSPHERE}

The optical impact of dust grains in Earth's atmosphere are long standing concerns for optical astronomy. The opacity can be measured by using small telescopes to monitor the brightness evolution of well known stars. Also, sensors which are exposed to the environment, can be affected by dust. For example, capacitative measurement techniques can be affected by dust grains ${ }^{4}$ and thereby compromise the telescope and potentially degrade the observation. The TMT site testing therefore monitored the dust content at the candidate sites.

\subsection{Instrumentation and setup}

The details of the TMT site monitoring systems are provided elsewhere*. What is noted here is that the site testing MASS-DIMM telescopes were mounted on towers bringing the telescopes to an approximate altitude of $7 \mathrm{~m}$ above each site. The base of the telescopes, below which the dust sensors were installed, were therefore approximately $6.5 \mathrm{~m}$ above the ground. To protect the sensors from the environmental conditions, they were installed inside a metal shelter which was attached to the top level of the tower, just below the telescope base. The sensor nozzle was reaching the outside of the shelter through an approximate $1 \mathrm{~cm}$ wide hole and was pointing downwards to prevent precipitation to enter the system. A night time image of a TMT tower hosting this instrumental setup is shown in Figure 1.

Commercially available two channel devices by MetOne (model GT-521) were used as dust sensors. Those

\footnotetext{
*sitedata.tmt.org
} 


\begin{tabular}{c|ccc} 
Site & $\begin{array}{c}\text { First dust } \\
\text { measurement }\end{array}$ & $\begin{array}{c}\text { Last dust } \\
\text { measurement }\end{array}$ & $\begin{array}{c}\text { Total number of measuremnts } \\
\text { in 0.3 / 0.5 / 1 / / 5 } \mu \text { m channel }\end{array}$ \\
\hline Tolar & Feb.14, 2006 & Dec.02, 2006 & $48,087 / 43,499 / 40,968 / 39,143 / 31,403$ \\
Armazones & Feb.11, 2006 & Mar.09, 2008 & $72,736 / 72,195 / 72,052 / 70,313 / 55,494$ \\
Tolonchar & Apr.04, 2006 & Mar.09, 2008 & $68,839 / 96,980 / 97,017 / 96,904 / 92,311$ \\
San Pedro Mártir & May 04, 2006 & Jun.17, 2007 & $46,234 / 81,023 / 80,946 / 80,733 / 61,268$ \\
13 N & Nov.30, 2005 & Dec.03,2007 & $40,772 / 52,227 / 52,183 / 49,560 / 18,793$
\end{tabular}

Table 1. Log of the dust measurements collected at the TMT candidate sites. The measurements continued at some sites for several months more, however, those data were not included in this assessment.

sensors have one fixed channel which always records particles of $0.3 \mu \mathrm{m}$ in size. The second channel can be selected to measure the concentration of particles of either $0.3,0.5,1.0,2.0$ and $5.0 \mu \mathrm{m}$ size simultaneous with the first channel. A single measurement of both channels typically took approximately one minute. The employed measurement strategy was to cycle through the different size bins of the second channel and taking with each of them a simultaneous measurement of the $0.3 \mu \mathrm{m}$ particles in the first channel. This resulted in a measurement for each of the four larger size bins every six minutes, while obtaining five measurements of the $0.3 \mu \mathrm{m}$ bin in the same time period which were averaged in the analysis process. The times covered by dust measurements at each site are listed in Table 1. Before the deployment at the sites a careful cross comparison was conducted in the laboratory, showing that these sensors agreed typically to within $10 \%$.
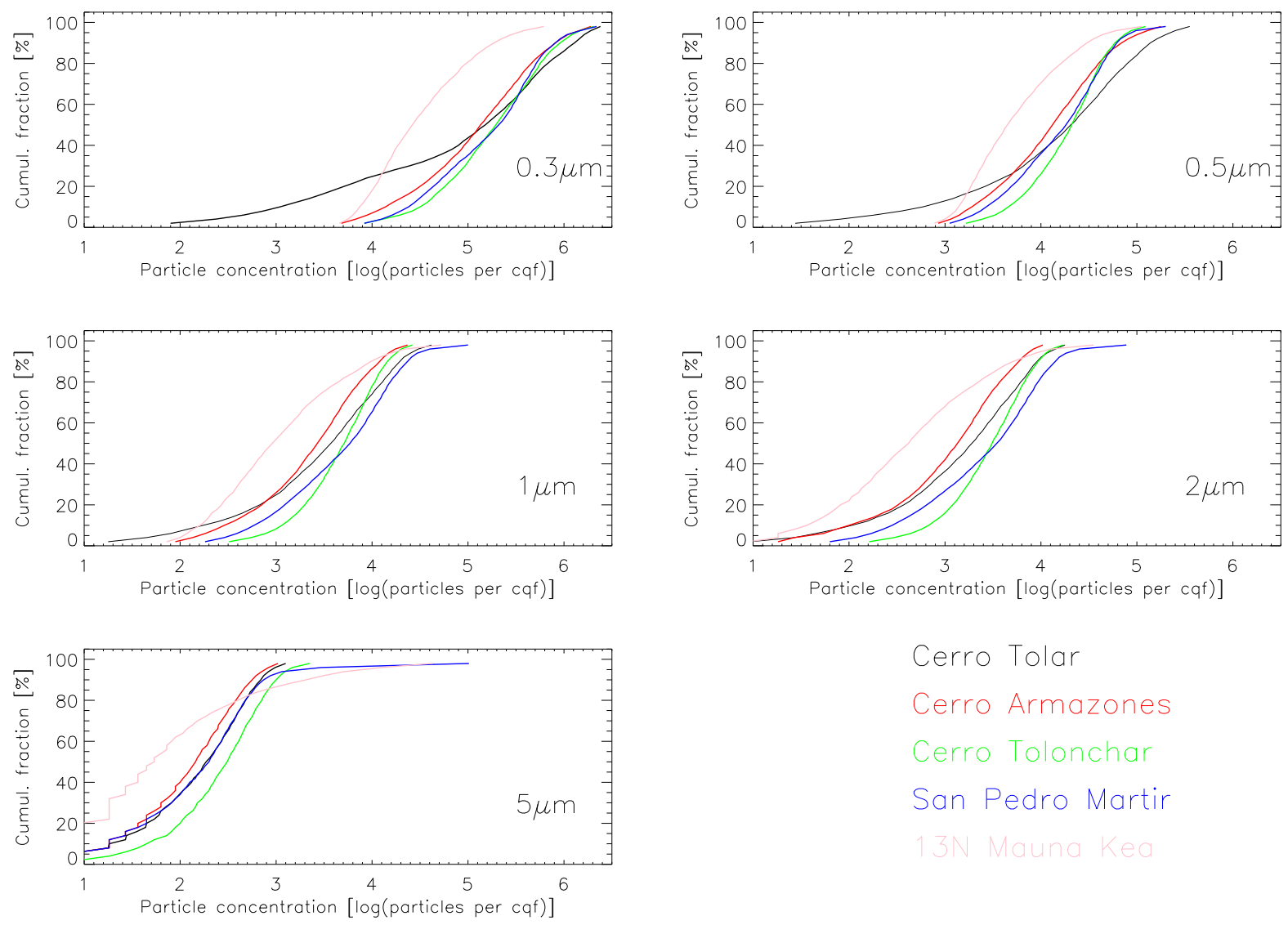

Figure 2. (color online) Overall cumulative statics of the dust measurements at the TMT candidate sites. Each panel represents a particle size bin, which are indicated. 

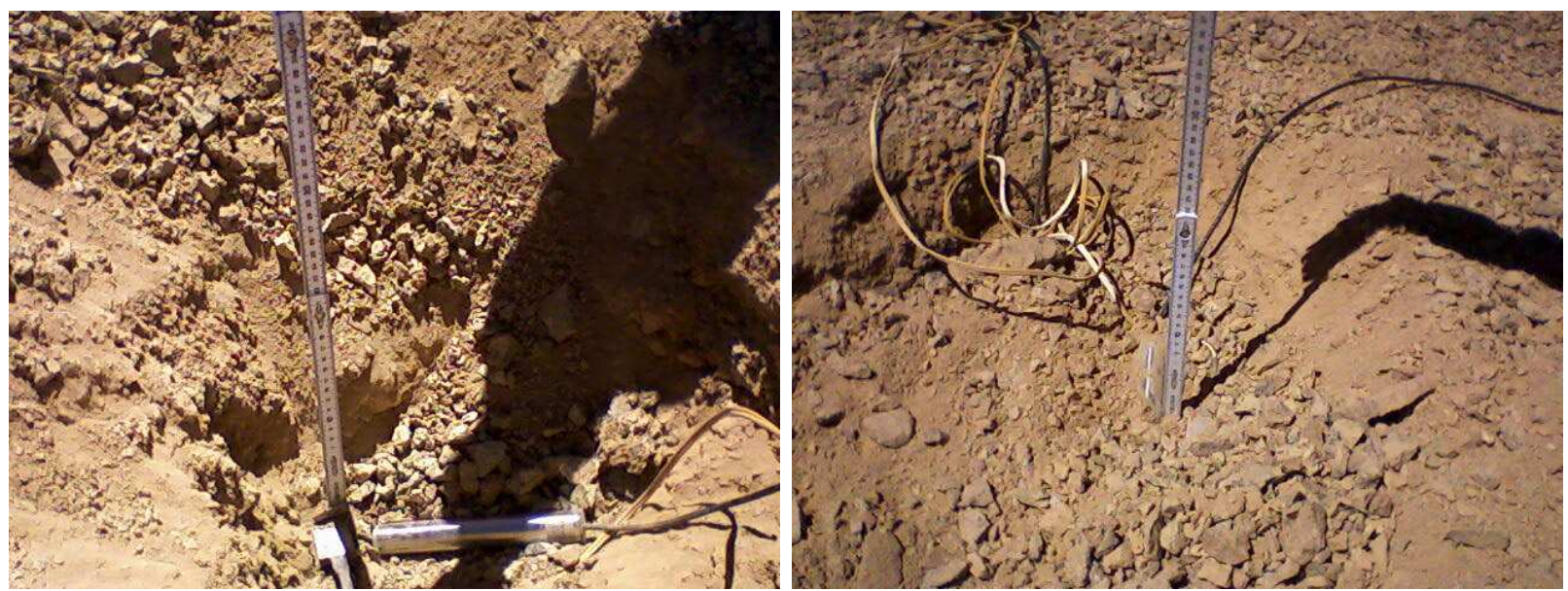

Figure 3. (color online) Pictures of the senors during deployment. Left panel: The sensors installed at $37 \mathrm{~cm}( \pm 1 \mathrm{~cm})$ depth. The tube of the soil temperature sensor and the heat flux plate are well visible. Right panel: At $16 \mathrm{~cm}( \pm 1 \mathrm{~cm})$ below the surface another set of sensors was installed, directly above the deep sensors.

\subsection{Results}

The statistics of the dust concentration as measured at the various sites are shown in Figure 2 . It can be seen that the lowest dust concentration was observed at $13 \mathrm{~N}$. This is understandable because that area of the Mauna Kea summit is a rocky plateau with little dust on its surface.

The Chilean sites show in general a higher dust concentration as they are located in the Atacama desert. Interestingly, it is Cerro Tolonchar which shows on average a higher concentration. It is probably the occurrence of the inversion layer at the altitude of the summit above the Salar de Atacama area which drives this increased level of dust concentration. Maybe this is in part also related to the presence of some flora in the summit area. Cerro Tolar on the other hand shows a very wide distribution of occurrences. As the summit area of that mountain is somewhat disturbed, fine dust which is otherwise buried under the surface "crust" can be stirred up by wind. The summit area at Cerro Armazones shows overall the lowest dust content across all particle sizes of the Chilean sites. Its terrain appears less disturbed and at the same time the visual impression of the soil material shows a lower content of fine dust as compared to Tolar.

San Pedro Mártir shows a dust concentration on a similar level to the Chilean sites. The presence of trees and overall flora at this site is suspected to be the reason for this dust concentration.

As also meteorological parameters were recorded at the sites, a number of correlations between dust occurrences and the meteorological features were also investigated. The findings of these studies will be reported elsewhere.

\section{SOIL ENERGY PROPERTIES}

The flow of energy through the soil of a site is an important factor in the strength of the ground layer optical turbulence. To compute the energy available to drive night time atmospheric turbulence, the energy accumulated during the day and the heat capacity and heat transfer properties of the soil must be known. For numerical models these factors are commonly obtained from large scale data bases based on various measurements and soil models. ${ }^{7}$ While such models are probably sufficient for typical conditions when averaging takes place on a larger spatial scale, i.e., non-zero or not too low wind speeds, as for very low wind speeds the properties of the local terrain will become of greater importance. ${ }^{8}$ Only in the case of Cerro Armazones was it possible to set up instrumentation to at least obtain first measurements of the soil heat conduction coefficient at the summit of that mountain. A similar study had been conducted by the ALMA site project, ${ }^{9}$ based on measurements at several more depths than done here but based entirely on measurements of the soil temperature at those depths alone. 

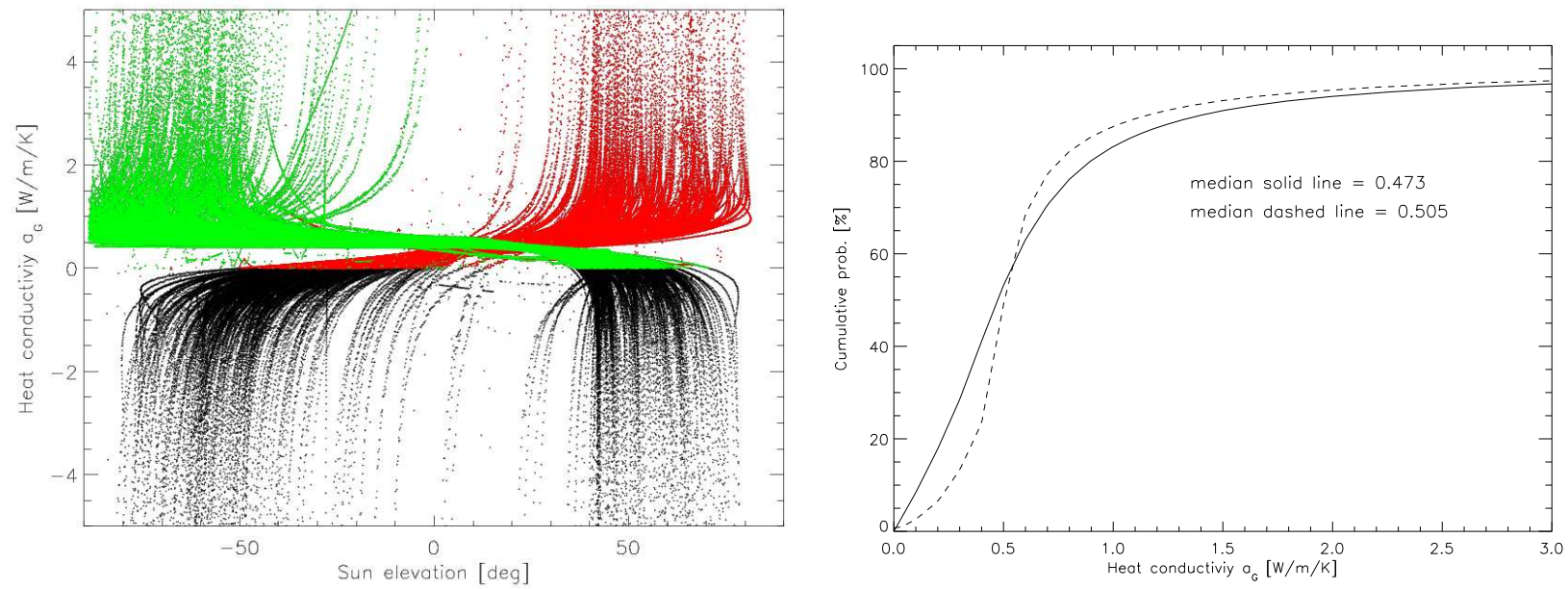

Figure 4. (color online) Left panel: The measured heat conduction coefficient $a_{G}$ in dependence of the sun elevation on Cerro Armazones. The red points represent 107, 461 measurements when the temperature gradient $T\left(z_{1}\right)-T\left(z_{2}\right)$ and both heat fluxes $Q\left(z_{1}\right), Q\left(z_{2}\right)$ are positive, while the green points show 185, 749 measurements when those gradients are negative. Overall 49,372 measurements showed mixed gradients which are marked by the black points. Right panel: The cumulative statistics of the heat conduction coefficient measurements from the left panel. The solid line represents the statistics based on positive gradients (red points in the left panel), while the dashed line is based on the negative ones (green points in the left panel).

\subsection{Instrumentation and setup}

The deployed instrumentation consisted of two pairs of ground heat flux plates and soil temperature sensors. Both sensors were commercially available products; the heat flux plates (model CN3) are manufactured by Middleton and the soil temperature sensor (model TS-1) which is made by MonitorSensors. A pair of such sensors was used at each TMT candidate site for several years in order to allow for the proper calibration of the acoustical turbulence sensors SODAR ${ }^{(10,11)}$. However, only after the decommissioning of the station from Cerro Tolonchar in late 2008, did a pair of sensors become available to allow the installation of two pairs of sensors at a single site, i.e., Cerro Armazones. Each pair - one temperature and one heat flux sensor - was buried in the ground at different depths, but on top of each other. During the installation on January 27, 2009, care was taken to embed the sensors into soil corresponding to the respective depths, i.e., not mixing material from the upper into the lower layer and vice versa. Images of the setup are shown in Fig. 3. Due to the slope of the terrain the depth of each sensor could only be determined to approximately $1 \mathrm{~cm}$. The recording of data once per minute commenced on February 3, 2009 and ended on October 11, 2009.

\subsection{Preliminary results for the heat conduction coefficient}

The heat conduction coefficient $a_{G}$ of the soil at the summit of Armazones was estimated using the basic definition

$$
Q_{G}=a_{G} \frac{\partial T}{\partial z}
$$

where $Q_{G}$ is the ground heat flux and $T$ is the soil temperature at various depths $z$ (in this case there two depths). The average conduction $\left\langle a_{G}\right\rangle$ in the slab of soil between the sensors at $z_{1}$ and $z_{2}$ is obtained from the measurements by

$$
<a_{G}>=0.5 \cdot\left(Q_{G, z 1}+Q_{G, z 2}\right) \cdot \frac{z_{1}-z_{2}}{T_{1}-T_{2}} .
$$

Due to the large vertical separation of the sensors and the proximity of one pair of sensors to the surface, there are numerous occasions under which the directions of the temperature gradient and heat fluxes were inverted. Under such conditions the determination of the averages is misleading and only measurements were selected when both, the temperature gradient $\left(T_{z 1}-T_{z 2}\right)$, and both heat flux measurements, were either positive or negative. 
To give a visual impression of the data, in the left panel of Fig. 4 the determined $a_{G}$ values as function of the sun elevation are shown. The largest scatter of the values is seen under large absolute sun elevation angles. In the Armazones region wind speeds typically drop during the last few hours before sun set, and at the begin of the night the heat flow at both investigated depths is still directed downwards. Therefore, Fig. 4 indicates that the more stable conditions under which the conductivity is probably measured best is around sun set and rise in this area.

The statistics of the so determined $a_{G}$ values are shown in the right panel of Fig. 4. With the medians of those distributions being close to $0.5 \mathrm{Wm}^{-1} \mathrm{~K}^{-1}$ the value found is slightly larger than the commonly given ${ }^{12}$ value for dry sand of $0.3 \mathrm{Wm}^{-1} \mathrm{~K}^{-1}$. This can be explained by the presence of small rocks and stones in the measured volume (see Fig. 3), as for rock/granite $a_{G}=2.73 \mathrm{Wm}^{-1} \mathrm{~K}^{-1}$ is found in the literature. ${ }^{12}$ The summit soil of Armazones (as well as at most of the Atacamenian summits) is composed of such a mix of dust, sand and rock, with the mountain main rock appearing at greater depths than were reached here. Also, as no other filtering of the data was applied than for times when heat flow and temperature gradients were aligned, the measurements are probably affected by seasonal variations of the humidity of the soil.

\section{SUMMARY}

The dust measurements of the TMT site testing campaign have been presented. Overall it was found that the dust concentration in the air is lowest at the most rocky and basically vegetation free site of the sample, i.e., $13 \mathrm{~N}$ on Mauna Kea. This holds for all measured particle sizes. The three investigated Chilean sites and San Pedro Mártir show dust concentration at a similar level, with Cerro Armazones showing the lowest average concentrations. These differences appear not unexpected as the dust concentration is expected to correlate with the geological and vegetational conditions at the sites. It is interesting to note that Cerro Tolonchar, which is an isolated summit with little vegetation but significantly more than the other Chilean sites, shows the highest of level dust concentration. This could also be due to the particular meteorological conditions at this location.

Also, preliminary results of an experiment to assess the soil heat conduction coefficient at Cerro Armazones were reported. The value found for the heat conduction coefficient $\left\langle a_{G}\right\rangle=0.5 \mathrm{Wm}^{-1} \mathrm{~K}^{-1}$ represents an average value for the autumn/winter/spring period at the Cerro Armazones summit. The difficulty of measuring in-situ the energetic properties of the soil has become obvious and a further analysis of those measurements should allow us to assess the ground heat capacity and diffusivity as well.

Using such in-situ measurements as input to numerical models of the air flow above the sites, are expected to improve the models, in particular under conditions when the contact time of the air and the soil is large, i.e., under low wind speeds.

\section{ACKNOWLEDGMENTS}

The TMT Project gratefully acknowledges the support of the TMT partner institutions. They are the Association of Canadian Universities for Research in Astronomy (ACURA), the California Institute of Technology and the University of California. This work was supported as well by the Gordon and Betty Moore Foundation, the Canada Foundation for Innovation, the Ontario Ministry of Research and Innovation, the National Research Council of Canada, the Natural Sciences and Engineering Research Council of Canada, the British Columbia Knowledge Development Fund, the Association of Universities for Research in Astronomy (AURA) and the U.S. National Science Foundation.

\section{REFERENCES}

[1] Sanchez S.F., Aceituno J., Thiele U., et al., "The Night Sky at the Calar Alto Observatory", 2007, PASP, 119,1186

[2] Stalin C.S., Hegde M., Sahu D.K., et al., "Night sky at the Indian Astronomical Observatory during 20002008", 2008, Bull. of the Astron. Soc. of India, 36, 2, 111-127

[3] Mayer U., "Astronomical Site Testing in South Africa", 1967, ESO Publication

[4] Buous S., Menzies J., Gajjar H., "SALT segmented primary mirror: commissioning capacitive edge sensing system and performance comparison with inductive sensor", 2008, SPIE, 7012, 7012-3G 
[5] Bounhir A., Benkhaldoun Z., El Arbi Siher, Lazrek M., "ELT site prospect in Morocco: aerosol characterization", 2006, SPIE, 6269, 6269-2D

[6] Schöck M., Els, S., Riddle R., Skidmore W., Travouillon T., et al., "Thirty Meter Telescope Site Testing I: Overview ", 2009, PASP, 121, 384

[7] McNider R.T., Lapenta W.M., Biazar A.P., et al., "Retrieval of Model Grid-Scale Heat Capacity Using Geostationary Satellite Products. Part I: First Case-Study Application", 2005, Journal of Appl. Meteorology, 44,1346

[8] Els S.G., Vogiatzis K., "TMT studies on the influence of short term changes of local thermodynamic conditions on the seeing: CFD vs. observations", 2006, SPIE, 6267, 67E

[9] Snyder L.A., Radford S.J.E., Holdaway M.A., "Underground Temperature Fluctuations and Water Drainage at Chajnantor", ALMA Memo 314, NRAO, 2000 June 30

[10] Travouillon T., Schöck M., Els S., Riddle R., Skidmore W., "Using a Sodar to Measure Optical Turbulence and Wind Speed for the Thirty Meter Telescope Site Testing. Part I: Reproducibility" 2011, Boundary-Layer Meteorology, 141, 2, 273

[11] Travouillon T., Schöck M., Els S., Riddle R., Skidmore W., "Using a Sodar to Measure Optical Turbulence and Wind Speed for the Thirty Meter Telescope Site Testing. Part II: Comparison with Independent Instruments", 2011, Boundary-Layer Meteorology, 141, 2, 289

[12] Stull R.B., "An Introduction to Boundary Layer Meteorology", 1988, Kluver Academic Publishers 